\title{
Boundary Migration in Rutile
}

\author{
Shelley R. Gilliss, N. Ravishankar ${ }^{1}$, Jeffrey K. Farrer² and C. Barry Carter
}

Dept. of Chemical Engineering and Materials Science, University of Minnesota

421 Washington Ave. S.E., Minneapolis MN 55455 USA

${ }^{1}$ Now at the Indian Institute of Science, Bangalore, India

${ }^{2}$ TSL/EDAX, Draper, Utah, 84020

$\mathrm{TiO}_{2}$ is a vital material in several technologies including, photocatalysis, gas sensing, biomaterials and optical coatings. Among the several crystal structures of this oxide, rutile has the highest density and microhardness, the highest index of refraction and the highest temperature stability [1]. The processing of dense polycrystalline materials often includes the addition of a liquid-forming phase at higher temperatures. This technique is known as liquid-phase sintering and has been studied extensively [2,3]. Rutile boundaries containing an amorphous phase have been used to study boundary migration and grain-boundary grooving.

Visible-light (VLM), scanning electron (SEM) and transmission electron microscopy (TEM) in addition to electron-backscatter diffraction (EBSD) and a focused-ion beam (FIB) tool were used to characterize boundary migration in rutile. EBSD analysis was carried out on a Philips XL30 FEG SEM equipped with a DigiView 1612 high-resolution, high-speed CCD camera. A $2.5 \mathrm{~cm}$ sampleto-camera distance was used and $\sim 70^{\circ}$ sample tilt. A Philips CM30 operated at $300 \mathrm{kV}$ was used for TEM characterization and an FEI DB235 was used for FIB work. Pulsed-laser deposition (PLD) has been used to deposit thin films $(\sim 100 \mathrm{~nm}$ thick) of silica glass on single-crystals of rutile. The film/substrate assembly is then fabricated into bicrystals of known boundary-plane orientation by hot pressing. Bicrystals were fabricated with boundary planes of nominal surface orientation of (001) and (110). After diffusion bonding a surface perpendicular to the interface is cut and polished. Bicrystals are then heat treated in air at $1650^{\circ} \mathrm{C}$ for varying lengths of time. Figure 1 is a VLM image of a rutile bicrystal which as been heat treated for 4 hours. During this heat treatment migration of the boundary initiates at parallel grooves contained in the crystal on the right-hand side. EBSD analysis shows that this parallel set of grooves is due to the presence of $3^{\circ}$ boundaries within this crystal. While the boundary migrates from the left to the right with respect to the image, the $3^{\circ}$ boundaries propagate into the reprecipitated rutile. The (001) boundary plane and (110) free surface grow at the expense of the (110) boundary plane and (001) free surface (free surface determined by EBSD). A FIB was used to prepare a cross-section TEM specimen at the migrated boundary. The FIB section was taken from an area in which the migrated boundary was $40 \mu \mathrm{m}$ from the groove remnant. A wide and shallow groove is formed at this migrated boundary and is illustrated in the bright-field (BF) TEM image in Figure 2. Of particular interest in this image is the nearly $120^{\circ}$ groove at the free surface (see schematic in Figure 3) and the location of the grain boundary. Often in EBSD analysis and grain-boundary grooving work, grain boundaries are assumed to have a perpendicular intersection to the free surface. This FIB-prepared specimen shows in this particular case, this assumption does not hold true. Due to the grooving at the free surface and therefore a reduction in energy at the free surface, the speed of migration is faster away from the surface. Figure 3 is a schematic of this migrated boundary. The original boundary consisted of (110) and (001) planes. The side with the (001) initial boundary plane migrates into the other crystal. A benefit of using a site-specific specimen preparation technique such as FIB allows for the grain-boundary profile to accurately be determined.

A variety of microscopy techniques has been used to study boundary migration in rutile. Migration in this case has originated at low-angle boundaries. The groove formed at the free surface slows the rate of migration at the surface. FIB allows the profile of the groove to be determined accurately.

References

1. J. Szczyrbowski, et al., Surf. Coat. Tech., 112 (1999) 261.

2. N. Ravishankar and C.B. Carter, Microsc. Microanal., 5 (Suppl. 2) (1999) 800. 
3. N. Ravishankar, H. Schmalzried, and C.B. Carter, Microsc. Microanal., 5 (1999) (Suppl. 2) 812.

4. The authors would like to thank Joe Michael (Sandia National Laboratories) for preparing FIB specimens, Velimir Radmolicov and Eric Stach (Lawrence Berkeley National Laboratory) for access and assistance to the FIB. This work is supported by the U.S. Department of Energy under grant DE-DE-FG02-01ER45883.

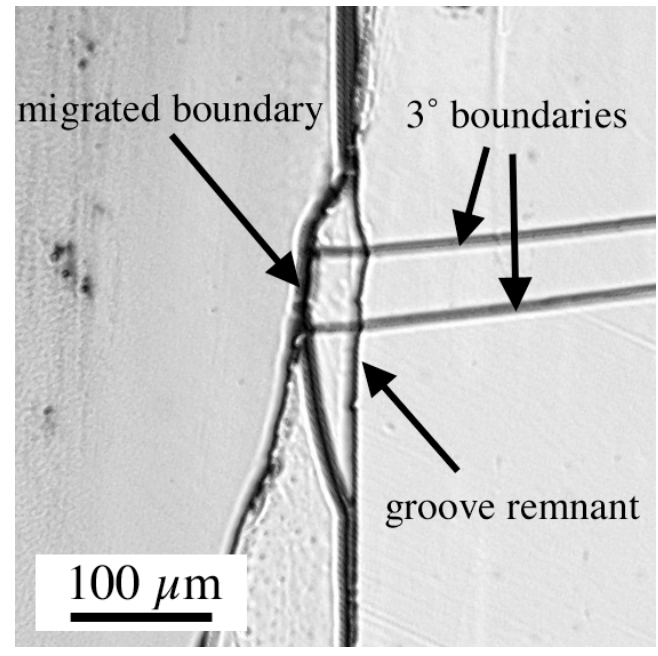

Fig.1. VLM image of migrated grain boundary in a rutile bicrystal. Migration originates at low-angle, $3^{\circ}$ boundaries, contained in the crystal on the right and propagate into the migrated region.

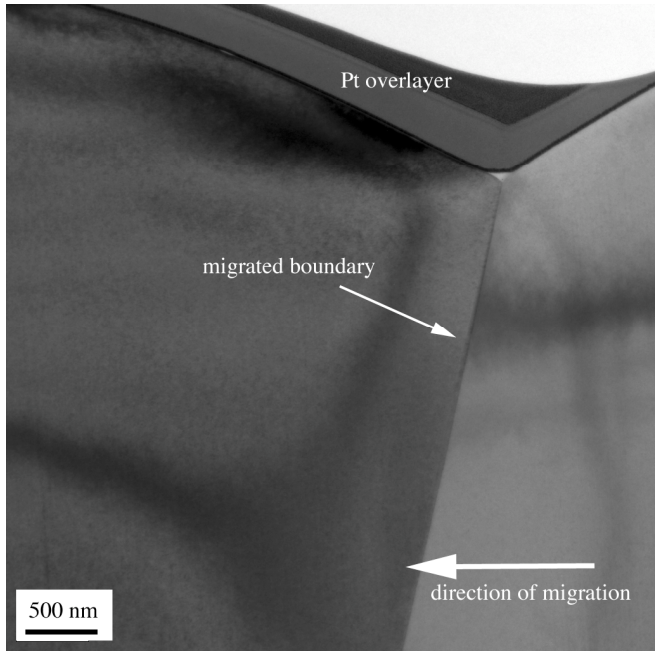

Fig. 2. BF TEM image of a FIB section taken at a migrated boundary in rutile. Due to the grooving process, which lowers the boundary energy at the surface, the migration progresses faster away from the free surface.

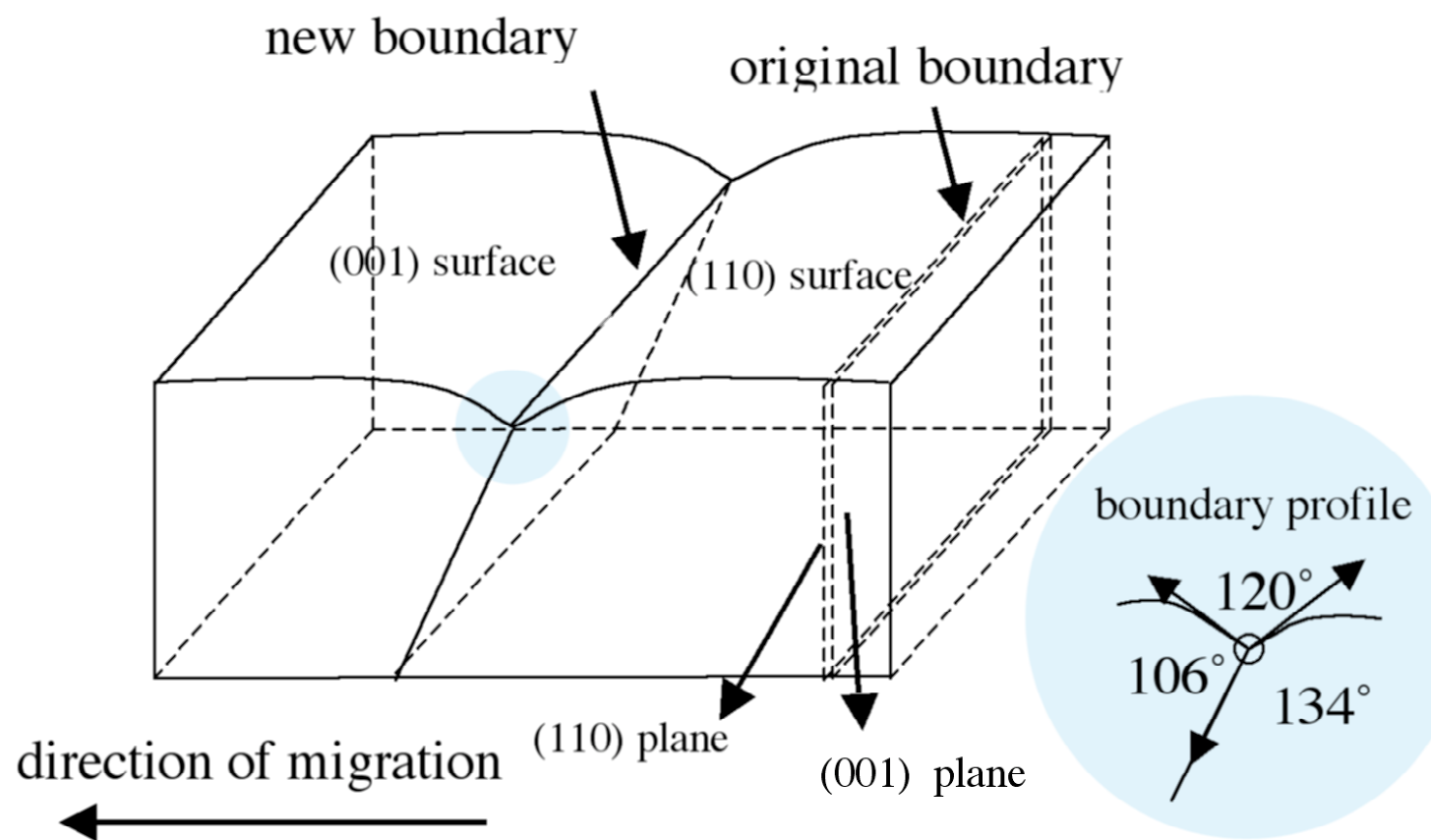

Fig. 3. Schematic of migrated boundary in rutile. The boundary profile can be measure accurately from the TEM image. 